\title{
Analysis of the small oscillations of a system viscous liquid- barotropic gas in a container
}

\author{
D. Vivona 1 - P. Capodanno
}

Received: 26 June 2016/Accepted: 12 May 2017/Published online: 14 June 2017

(C) Springer Science+Business Media Dordrecht 2017

\begin{abstract}
The authors study the problem of the small oscillations of a system viscous liquid- barotropic gas in a fixed container. From the equations of motion and using two auxiliary problems, they reduce the problem to a system of two operatorial equations in a suitable hilbertian space. By means of the methods of the functional analysis, they prove the existence of the spectrum and study its properties.
\end{abstract}

Keywords Viscous fluid · Barotropic gas · Small oscillations · Variational - Operatorial and spectral methods

\section{Introduction}

The problem of the small oscillations of an incompressible inviscid liquid in a container by means of the methods of the functional analysis was a subject of a pioneering work by Moiseyev [1] and has been extensively studied afterwards. We can find these

\footnotetext{
D. Vivona $(\bowtie)$

Department of Basic and Applied Sciences for Engineering, Faculty of Civil and Industrial Engineering, "Sapienza" University of Rome, Rome, Italy e-mail: doretta.vivona@uniroma1.it

P. Capodanno

University of Franche-Compté, Besançon, France

e-mail: pierre.capodanno@neuf.fr
}

works in the books [2-4]. The more difficult case of the viscous fluid has been also the subject of many papers, see [5].

The problem of the small oscillations of a system homogeneous inviscid liquid-gas has been studied in the work [6], that is presented in the book [3], but with simplified equations for the gas. The case of heterogeneous inviscid liquid with the general equations for the gas was treated in [7].

In this work, the authors study the problem of the small oscillations of a system viscous liquid-barotropic gas.

At first, they obtain the equations of motion of the system, using the general equations for the gas. Introducing two auxiliary problems that generalize the problems considered in $[3,6]$, they replace the equations and the boundary conditions for the gas by an operatorial equation and can reduce the problem to two operatorial equations in a suitable hilbertian space.

They prove the existence of the spectrum formed by zero, that is an eigenvalue with infinite multeplicity and by isolated eigenvalues, with real part positive, so that the system is stable and they show that there exists a set of positive real eigenvalues having zero as point of accumulation.

Finally, they reduce the problem to a Krein-Langer quadratic pencil [8], so that the non real eigenvalues can have as only one point of accumulation: the infinity. 


\section{Position of the problem}

In the equilibrium position, the viscous liquid (resp. the gas) occupies a domain $\Omega$ (resp. $\Omega_{0}$ ) bounded by a part $\Sigma$ (resp. $\Sigma_{0}$ ) of the wall of the container and by horizontal interface $\Gamma$, the equation of which with respect to the orthogonal axes $O x_{1} x_{2} x_{3}\left(O x_{3}\right.$ vertical directed upwards) is $x_{3}=0$.

Their position at the instant $t$ is modified by the index $t$, example $\Gamma_{t}$.

We denote by $g$ the constant acceleration due to the gravity.

We want to study the small oscillations of this system about its equilibrium position, obviously in linear theory.

\section{Equations of motion}

\subsection{Viscous liquid}

Let $x_{3}$ be the unit vector in the $x_{3}$ direction, vertical upwards.

If $\rho$ is the constant density of the liquid, and $\mathcal{P}^{e}$ its pressure in equilibrium position,

$$
\mathcal{P}^{e}=-\rho g x_{3},
$$

we denote by $\mu$ its constant viscosity coefficient, $p=$ $\mathcal{P}-\mathcal{P}^{e}$ the dynamic pressure (the difference between the pressure $\mathcal{P}$ and $\left.\mathcal{P}^{e}\right)$ and $\boldsymbol{u}\left(x_{1}, x_{2}, x_{3}, t\right)$ the (small) displacement of a particle of the liquid with respect to its equilibrium position, the equations of motion are:

$\rho \ddot{\boldsymbol{u}}=-\operatorname{grad} p+\mu \Delta \dot{\boldsymbol{u}}$,

(Navier - Stokes equation) in $\Omega$,

div $\dot{\boldsymbol{u}}=0$ (incompressibility) in $\Omega$,

or, after integration between the datum of the equilibrium and $t$ :

$\operatorname{div} \boldsymbol{u}=0$ in $\Omega$,

and the kinematic condition on $\Sigma$

$\left.\boldsymbol{u}\right|_{\Sigma}=0$

\subsection{Gas}

If $\rho_{0}^{*}\left(x_{1}, x_{2}, x_{3}, t\right)$ and $p_{0}^{*}\left(x_{1}, x_{2}, x_{3}, t\right)$ are the density and the pressure of the gas, we have the state equation

$p_{0}^{*}=P\left(\rho_{0}^{*}\right)$

where $P$ is a given smooth increasing function.

The density $\rho_{0}$ and the pressure $p_{0}$ in the equilibrium position must verify the Eq. (4) and the equilibrium equation

$\frac{1}{\rho_{0}} \operatorname{grad} p_{0}=-g \boldsymbol{x}_{3}$,

so that $\rho_{0}$ and $p_{0}$ are function of $x_{3}$ with

$\frac{d p_{0}}{d x_{3}}=-\rho_{0}\left(x_{3}\right) g$

or, if $p_{0}(0)=0$,

$p_{0}\left(x_{3}\right)=-\int_{0}^{x_{3}} \rho_{0}(w) g d w$.

The equations of the motion of the gas are (4) and, if $\boldsymbol{u}_{0}\left(x_{1}, x_{2}, x_{3}, t\right)$ is the (small) displacement of a particle with respect to its equilibrium position,

$\rho_{0}^{*} \ddot{\boldsymbol{u}}_{0}=-\rho_{0}^{*} g \boldsymbol{x}_{3}-\boldsymbol{g r a d} p_{0}^{*}($ Euler's equation $)$,

$\frac{\partial \rho_{0}^{*}}{\partial t}+\operatorname{div}\left(\rho_{0}^{*} \dot{\boldsymbol{u}_{0}}\right)=0$ (continuity equation).

The kinematic conditions on $\Sigma_{0}$ are, after integration,

$\left.\boldsymbol{u}_{0} \cdot \boldsymbol{n}\right|_{\Sigma_{0}}=0$

$\left.u_{n 0}\right|_{\Gamma}=\left.u_{n}\right|_{\Gamma}=0$,

where $\boldsymbol{n}$ is the unit vector normal to $\Gamma$, directed upwards.

Since the volumes of the liquid and of the gas are constant, we get:

$\left.\int_{\Gamma} u_{n}\right|_{\Gamma} d \Gamma=0 ;\left.\int_{\Gamma} u_{0 n}\right|_{\Gamma} d \Gamma=0$. 
3.3 Boundary conditions on the moving interface $\Gamma_{t}$

If we denote by $\sigma_{i j}$ (resp. $\varepsilon_{i j}$ ) the stress (resp.strain) tensor of the viscous liquid, we must have

$\sigma_{i j} n_{j}=-p_{0}^{*} n_{i}$ on $\Gamma_{t}$,

or, if $\mathcal{P}=-\rho g x_{3}+p+\cdots$ is the pressure of the liquid,

$\left(-\mathcal{P} \delta_{i j}+2 \mu \varepsilon_{i j}\right) n_{j}=-p_{0}^{*} n_{i}$ on $\Gamma_{t}$.

Introducing the dynamic pressure $\hat{p}_{0}^{*}$ of the gas by

$p_{0}^{*}=p_{0}\left(x_{3}\right)+\hat{p}_{0}^{*}+\cdots=-\int_{0}^{x_{3}} \rho_{0}(w) g d w+\hat{p}_{0}^{*}+\cdots$,

we obtain

$\left[-\int_{0}^{x_{3}} \rho_{0}(w) g d w+\left.\hat{p}^{*}\right|_{\Gamma}+\left.\rho g u_{n}\right|_{\Gamma}-\left.p\right|_{\Gamma}\right] n_{i}$

$+2 \mu \varepsilon_{i j} n_{j}=0$ on $\Gamma$

and, consequently,

$\left.\varepsilon_{13}\right|_{\Gamma}=0,\left.\quad \varepsilon_{23}\right|_{\Gamma}=0$,

$\left.\hat{p}_{0}^{*}\right|_{\Gamma}-\left.p\right|_{\Gamma}+\left.\left(\rho-\rho_{0}(0)\right) g u_{n}\right|_{\Gamma}+\left.2 \mu \varepsilon_{33}\right|_{\Gamma}=0$.

3.4 Transformation of the equations of the motion of the gas

We set, besides $p_{0}^{*}=p_{0}\left(x_{3}\right)+\hat{p}_{0}^{*}\left(x_{1}, x_{2}, x_{3}, t\right)+\cdots$,

$$
\rho_{0}^{*}=\rho_{0}\left(x_{3}\right)+\hat{\rho}_{0}^{*}\left(x_{1}, x_{2}, x_{3}, t\right)+\cdots,
$$

where $\hat{p}_{0}^{*}$ and $\hat{\rho}_{0}^{*}$ are of the first order with respect to amplitude of the oscillations; we use the method presented in ([9], pp. 62-64).

From (4) and (6) and setting classically $c_{0}^{2}\left(x_{3}\right)=$ $P^{\prime}\left(\rho_{0}\left(x_{3}\right)\right)$, we obtain

$\hat{\rho}_{0}^{*}=-\operatorname{div}\left(\rho_{0}\left(x_{3}\right) \boldsymbol{u}_{0}\right)$,

$\hat{p}_{0}^{*}=-c_{0}^{2}\left(x_{3}\right) \operatorname{div}\left[\rho_{0}\left(x_{3}\right) \boldsymbol{u}_{0}\right]$

and, using the Euler's equation (5),

$$
\ddot{\boldsymbol{u}}_{0}=\operatorname{grad}\left[\frac{c_{0}^{2}\left(x_{3}\right)}{\rho_{0}\left(x_{3}\right)} \operatorname{div}\left[\rho_{0}\left(x_{3}\right) \boldsymbol{u}_{0}\right]\right] \text {. }
$$

In conditions of the Lagrange's theorem, we introduce the displacement potential $\varphi_{0}$ defined by

$\boldsymbol{u}_{0}=\operatorname{grad} \varphi_{0}$

so the (13) can be rewritten

$\ddot{\varphi}_{0}=\frac{c_{0}^{2}\left(x_{3}\right)}{\rho_{0}\left(x_{3}\right)} \operatorname{div}\left[\rho_{0}\left(x_{3}\right) \operatorname{grad} \varphi_{0}\right]+C(t)$.

So, we have

$$
\begin{aligned}
\int_{\Omega_{0}} \rho_{0} c_{0}^{-2} \ddot{\varphi_{0}} d \Omega_{0}= & \int_{\Omega_{0}} \operatorname{div}\left(\rho_{0} \operatorname{grad} \varphi_{0}\right) d \Omega_{0} \\
& +C(t) \int_{\Omega_{0}} \rho_{0} c_{0}^{-2} d \Omega_{0} .
\end{aligned}
$$

From the Green formula and (7), (9), we have

$\int_{\Omega_{0}} \operatorname{div}\left(\rho_{0} \operatorname{grad} \varphi_{0}\right) d \Omega_{0}=0$

and then

$$
\int_{\Omega_{0}} \rho_{0} c_{0}^{-2} \ddot{\varphi_{0}} d \Omega_{0}=C(t) \int_{\Omega_{0}} \rho_{0} c_{0}^{-2} d \Omega_{0},
$$

$\varphi_{0}$ being determined up to an additional function of $t$; we can suppose that

$$
\varphi_{0} \in H_{\Omega_{0}}^{1}\left(\Omega_{0}\right) \stackrel{\text { def }}{=}_{=}^{\text {a }}\left\{\varphi_{0} \in H^{1}\left(\Omega_{0}\right) ; \int_{\Omega_{0}} \rho_{0} c_{0}^{-2} \varphi_{0} d \Omega_{0}=0\right\} .
$$

Then $C(t)=0$ and the equations of motion of the gas take the form

$\ddot{\varphi}_{0}=\frac{c_{0}^{2}}{\rho_{0}} \operatorname{div}\left[\rho_{0}\left(x_{3}\right) \operatorname{grad} \varphi_{0}\right]$,

$\left.\frac{\partial \varphi_{0}}{\partial n}\right|_{\Sigma_{0}}=0,\left.\frac{\partial \varphi_{0}}{\partial n}\right|_{\Gamma}=-\left.u_{n}\right|_{\Gamma}$.

Besides, we have, using (12):

$\hat{p}_{0}^{*}=-\rho_{0} \ddot{\varphi}_{0}$

and the dynamic condition (11) can be rewritten

$\left.p\right|_{\Gamma}=\left.\left(\rho-\rho_{0}(0)\right) g u_{n}\right|_{\Gamma}+\mathcal{P}_{\Gamma}\left[\left.2 \mu \varepsilon_{33}\right|_{\Gamma}-\left.\rho_{0}(0) \ddot{\varphi}_{0}\right|_{\Gamma}\right]$,

where $\mathcal{P}_{\Gamma}$ is the orthogonal projector of $L^{2}(\Gamma)$ on $\tilde{L}^{2}(\Gamma)=\left\{f \in L^{2}(\Gamma) ; \int_{\Gamma} f d \Gamma=0\right\}$. 


\section{An operatorial equation for the viscous liquid}

\subsection{Variational equation}

Let $\tilde{\boldsymbol{u}}\left(x_{1}, x_{2}, x_{3}\right)$ be a smooth function defined in $\Omega$ and satisfying div $\tilde{\boldsymbol{u}}=0$ and $\left.\tilde{\boldsymbol{u}}\right|_{\Sigma}=0$ and $\overline{\tilde{\boldsymbol{u}}}$ its conjugate. From the Eq. (1), multipling by $\overline{\tilde{\boldsymbol{u}}}$, we have

$\int_{\Omega} \rho \ddot{\boldsymbol{u}} \cdot \overline{\tilde{\boldsymbol{u}}} d \Omega=-\int_{\Omega} \operatorname{grad} p \cdot \overline{\tilde{\boldsymbol{u}}} d \Omega+\mu \int_{\Omega} \Delta \dot{\boldsymbol{u}} \cdot \overline{\tilde{\boldsymbol{u}}} d \Omega$.

Using the Green formula for the vectorial laplacian formula ([3], p. 128), we get

$$
\begin{aligned}
& \int_{\Omega} \rho \ddot{\boldsymbol{u}} \cdot \overline{\tilde{\boldsymbol{u}}} d \Omega=-2 \mu \int_{\Omega} \varepsilon_{i j}(\dot{\boldsymbol{u}}) \cdot \varepsilon_{i j}(\overline{\tilde{\boldsymbol{u}}}) d \Omega \\
& \quad+\left.\int_{\Gamma}\left(2 \mu \varepsilon_{33}-\left.p\right|_{\Gamma}\right) \overline{\tilde{u}}_{n}\right|_{\Gamma} d \Gamma
\end{aligned}
$$

and taking into account (16), the variational equation of the problem is:

$$
\begin{aligned}
& \int_{\Omega} \rho \ddot{\boldsymbol{u}} \cdot \overline{\tilde{\boldsymbol{u}}} d \boldsymbol{\Omega}+2 \mu \int_{\Omega} \varepsilon_{i j}(\dot{\boldsymbol{u}}) \cdot \varepsilon_{i j}(\overline{\tilde{\boldsymbol{u}}}) d \boldsymbol{\Omega} \\
& \quad+\left.\int_{\Gamma}\left[\left.\left(\rho-\rho_{0}(0)\right) g u_{n}\right|_{\Gamma}-\left.\mathcal{P}_{\Gamma} \rho_{0}(0) \ddot{\varphi}_{0}\right|_{\Gamma}\right] \overline{\tilde{u}}_{n}\right|_{\Gamma} d \Gamma=0,
\end{aligned}
$$

$\forall \tilde{\boldsymbol{u}}$ admissible.

\subsection{Operatorial equation}

We seek $\boldsymbol{u}$ in the space

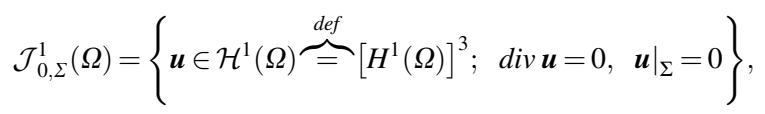

equipped with the scalar product

$$
(\boldsymbol{u}, \tilde{\boldsymbol{u}})_{1}=\int_{\Omega} 2 \varepsilon_{i j}(\boldsymbol{u}) \cdot \varepsilon_{i j}(\overline{\tilde{\boldsymbol{u}}}) d \Omega
$$

and we introduce the space

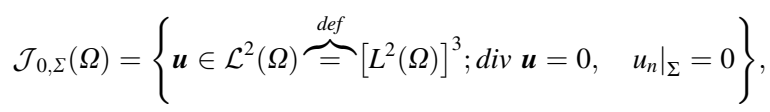

equipped with the scalar product of $\mathcal{L}^{2}(\Omega)$. For the meaning of the differential operators, we refer to ([3], n. 22).

It is well-known that the embedding $\mathcal{J}_{0, \Sigma}^{1}(\Omega) \subset$ $\mathcal{J}_{0, \Sigma}(\Omega)$ is continuous, dense and compact, see [3]. Let
$A$ be the unbounded operator of $\mathcal{J}_{0, \Sigma}(\Omega)$ associated to the pair $\left(\mathcal{J}_{0, \Sigma}^{1}(\Omega), \mathcal{J}_{0, \Sigma}(\Omega)\right)$ and to the scalar product $(\boldsymbol{u}, \tilde{\boldsymbol{u}})_{1}$.

We remark that, for each $\xi \in \tilde{L^{2}}(\Gamma)$, using a classical trace theorem in $\mathcal{H}^{1}(\Omega)$ :

$\left|\int_{\Gamma} \xi \overline{\tilde{u}}_{n}\right|_{\Gamma} d \Gamma|\leq c||\xi|_{\tilde{L}^{2}(\Gamma)}|| \tilde{\mathbf{u}} \|_{1}$,

so that there exists a bounded operator $T$ from $\tilde{L}^{2}(\Gamma)$ into $\mathcal{J}_{0, \Sigma}^{1}(\Omega)$ such as

$$
\left.\int_{\Gamma} \xi \overline{\tilde{u}}_{n}\right|_{\Gamma} d \Gamma=(T \xi, \tilde{\boldsymbol{u}})_{1} .
$$

Then, the variational Eq. (17) takes the form, setting $v=\frac{\mu}{\rho}$,

$$
\begin{aligned}
& \int_{\Omega} \ddot{\boldsymbol{u}} \cdot \tilde{\boldsymbol{u}} d \Omega+v(\dot{\boldsymbol{u}}, \tilde{\boldsymbol{u}})_{1}+\frac{1}{\rho}\left(T \left[\left.\left(\rho-\rho_{0}(0)\right) g u_{n}\right|_{\Gamma}\right.\right. \\
& \left.\left.\quad-\left.\mathcal{P}_{\Gamma} \rho_{0}(0) \ddot{\varphi}_{0}\right|_{\Gamma}\right], \tilde{\boldsymbol{u}}\right)_{1}=0, \forall \overline{\boldsymbol{u}} \in \mathcal{J}_{0, \Sigma}^{1}(\Omega) .
\end{aligned}
$$

We can replace it ([10], pp. 10-12) by the following: the operatorial equation is:

$$
\begin{aligned}
\ddot{\boldsymbol{u}}+ & v A \dot{\boldsymbol{u}}+\frac{1}{\rho} A T\left[\left.\left(\rho-\rho_{0}(0)\right) g u_{n}\right|_{\Gamma}-\left.\rho_{0}(0) \mathcal{P}_{\Gamma} \ddot{\varphi}_{0}\right|_{\Gamma}\right] \\
& =0, \forall \boldsymbol{u} \in \mathcal{J}_{0, \Sigma}^{1}(\Omega) .
\end{aligned}
$$

\section{Equations of the small oscillations of the system}

\subsection{Solutions}

We seek the solutions of the Eqs. (14), (15) and (18) that depend on time according to the law $\boldsymbol{u}(x, t)=$ $e^{-\lambda t} \boldsymbol{u}(x), \varphi_{0}(x, t)=e^{-\lambda t} \varphi_{0}(x), \lambda \in \mathbb{C}$. We obtain

$\lambda^{2} \boldsymbol{u}-\lambda v A \boldsymbol{u}+\frac{1}{\rho} A T\left[\left.\left(\rho-\rho_{0}(0)\right) g u_{n}\right|_{\Gamma}\right.$

$$
\left.-\left.\lambda^{2} \rho_{0}(0) \mathcal{P}_{\Gamma} \varphi_{0}\right|_{\Gamma}\right]=0,
$$

$\lambda^{2} \varphi_{0}=\frac{c_{0}^{2}}{\rho_{0}} \operatorname{div}\left[\rho_{0} \operatorname{grad} \varphi_{0}\right]$

$\left.\frac{\partial \varphi_{0}}{\partial n_{e}}\right|_{\Sigma_{0}}=0 \quad,\left.\quad \frac{\partial \varphi_{0}}{\partial n_{e}}\right|_{\Gamma}=-\left.u_{3}\right|_{\Gamma}$

$\left(\int_{\Omega_{0}} \rho_{0} c_{0}^{-2} \varphi_{0} d \Omega_{0}=0\right)$, 
where $\boldsymbol{n}_{e}$ is the unit vector normal to the boundary of $\Omega_{0}$, directed to the exterior of $\Omega_{0}$.

\subsection{Auxiliary problems}

We are going to replace the equations for $\varphi_{0},(20)$ and (21), by a single operatorial equation, using two auxiliary problems generalizing problems considered in [3].

\subsubsection{Problem I}

Let $u_{3} \in \tilde{L}^{2}(\Gamma)$. We shall find $\Phi_{01} \in H_{\Omega_{0}}^{1}\left(\Omega_{0}\right)$, generalized solution of the Neuman problem,

$\operatorname{div}\left[\rho_{0} \operatorname{grad} \Phi_{01}\right]=0$ in $\Omega_{0}$,

$\left.\frac{\partial \Phi_{01}}{\partial n}\right|_{\Sigma_{0}}=0,\left.\quad \frac{\partial \Phi_{01}}{\partial n}\right|_{\Gamma}=-u_{3}$.

It is easy to see that the variational formulation of this problem is

$$
\begin{aligned}
& \int_{\Omega_{0}} \rho_{0} \operatorname{grad} \Phi_{01} \cdot \operatorname{grad} \overline{\tilde{\Phi}}_{01} d \Omega_{0} \\
& \quad=-\int_{\Gamma} \rho_{0}(0) \overline{\tilde{\Phi}}_{01} u_{3} d \Gamma, \quad \forall \tilde{\Phi}_{01} \in H_{\Omega_{0}}^{1}\left(\Omega_{0}\right) .
\end{aligned}
$$

As the left-hand side is classically a scalar product in $H_{\Omega_{0}}^{1}\left(\Omega_{0}\right)$, the problem has only one solution by the Lax-Milgram theorem.

Therefore, we can set

$$
\Phi_{01}=T_{0} u_{3},
$$

where $T_{0}$ is a bounded operator from $\tilde{L}^{2}(\Gamma)$ into $H_{\Omega_{0}}^{1}\left(\Omega_{0}\right)$.

If $\gamma_{0}$ is the trace operator from $H^{1}\left(\Omega_{0}\right)$ into $L^{2}(\Gamma)$, we have

$$
\left.\Phi_{01}\right|_{\Gamma}=\gamma_{0} T_{0} u_{3} .
$$

Now, let $\tilde{\Phi}_{01}$ be the solution of the Problem I for the datum $\tilde{u}_{3} \in \tilde{L}^{2}(\Gamma)$; then we have easily

$$
\begin{gathered}
\int_{\Omega_{0}} \rho_{0} \operatorname{grad} \Phi_{01} \cdot \operatorname{grad} \overline{\tilde{\Phi}}_{01} d \Omega_{0}= \\
-\int_{\Gamma} \rho_{0}(0) u_{3} \mathcal{P}_{\Gamma}\left(\gamma_{0} T_{0} \overline{\tilde{u}}_{3}\right) d \Gamma .
\end{gathered}
$$

Now, we introduce

$$
C_{0}=-\rho_{0}(0) \mathcal{P}_{\Gamma} \gamma_{0} T_{0},
$$

which is an operator from $\tilde{L}^{2}(\Gamma)$ into $\tilde{L}^{2}(\Gamma)$. It is easy to see that $C_{0}$ is self-adjoint, positive definite and, like $\gamma_{0}$, compact.

\subsubsection{Problem II}

Let $f$ be an element of the space

$$
L_{\Omega_{0}}^{2}\left(\Omega_{0}\right) \overbrace{=}^{\text {def }}\left\{f \in L^{2}\left(\Omega_{0}\right) ; \int_{\Omega_{0}} \rho_{0} c_{0}^{-2} f d \Omega_{0}=0\right\},
$$

equipped with the scalar product

$$
(f, \tilde{f})_{L_{\Omega_{0}}^{2}\left(\Omega_{0}\right)}=\int_{\Omega_{0}} \rho_{0} c_{0}^{-2} f \overline{\tilde{f}} d \Omega_{0} .
$$

To find $\Phi_{02} \in H_{\Omega_{0}}^{1}\left(\Omega_{0}\right)$, generalized solution of the Neuman problem,

$$
\begin{aligned}
& -\operatorname{div}\left[\rho_{0} \operatorname{grad} \Phi_{02}\right]=\rho_{0} c_{0}^{-2} f \text { in } \Omega_{0}, \\
& \left.\frac{\partial \Phi_{02}}{\partial n_{e}}\right|_{\Gamma+\Sigma_{0}}=0,
\end{aligned}
$$

the variational formulation of this problem is

$$
\begin{aligned}
& -\int_{\Omega_{0}} \operatorname{div}\left(\rho_{0} \operatorname{grad} \Phi_{02}\right) \cdot \operatorname{grad} \overline{\tilde{\Phi}}_{02} d \Omega_{0} \\
& =\int_{\Omega_{0}} \rho_{0} c_{0}^{-2} f \overline{\tilde{\Phi}}_{02} d \Omega_{0}, \quad \forall \tilde{\Phi}_{02} \in H_{\Omega_{0}}^{1}\left(\Omega_{0}\right),
\end{aligned}
$$

and the problem has only one solution by the LaxMilgram theorem.

The embedding $H_{\Omega_{0}}^{1}\left(\Omega_{0}\right) \subset L_{\Omega_{0}}^{2}\left(\Omega_{0}\right)$ is classically continuous, dense and compact.

Let us call $A_{0}$ the unbounded operator of $L_{\Omega_{0}}^{2}\left(\Omega_{0}\right)$ associated to the pair $\left(H_{\Omega_{0}}^{1}\left(\Omega_{0}\right), L_{\Omega_{0}}^{2}\left(\Omega_{0}\right)\right)$ and to the scalar product

$$
\left(\Phi_{02}, \tilde{\Phi}_{02}\right)_{H_{\Omega_{0}}^{1}\left(\Omega_{0}\right)}=\int_{\Omega_{0}} \rho_{0} \operatorname{grad} \Phi_{02} \cdot \operatorname{grad} \overline{\tilde{\Phi}}_{02} d \Omega_{0} .
$$

The Eq. (25) can be rewritten

$$
\left(\Phi_{02}, \tilde{\Phi}_{02}\right)_{H_{\Omega_{0}}^{1}\left(\Omega_{0}\right)}=\left(f, \tilde{\Phi}_{02}\right)_{L_{\Omega_{0}}^{2}\left(\Omega_{0}\right)}, \forall \tilde{\Phi}_{02} \in H_{\Omega_{0}}^{1}\left(\Omega_{0}\right),
$$

so that we have

$$
A_{0} \Phi_{02}=f \text { or } \Phi_{02}=A_{0}^{-1} f .
$$




\subsubsection{General solution}

From (23) and (26), the solution of the Eqs. (20) and (21) is

$\varphi_{0}=\Phi_{01}+\Phi_{02}$,

taking $u_{3}=\left.u_{n}\right|_{\Gamma}$ and $f=-\lambda^{2} \varphi_{0}$.

Moreover,

$$
\begin{aligned}
\Phi_{02} & =A_{0}^{-1} f=-\lambda^{2} A_{0}^{-1} \varphi_{0} \\
& =-\lambda^{2} A_{0}^{-1}\left(T_{0} u_{3}-\Phi_{02}\right),
\end{aligned}
$$

and applying $A_{0}^{-1}$ :

$f=-\lambda^{2}\left(T_{0} u_{3}+A_{0}^{-1} f\right)$,

that is an unique equation replacing the Eqs. (20), (21) for the gas.

\subsection{Small oscillations}

Let us calculate the term $\left.\mathcal{P}_{\Gamma} \varphi_{0}\right|_{\Gamma}=\mathcal{P}_{\Gamma} \gamma_{0} \varphi_{0}$ of the Eq. (19). We obtain easily

$$
\begin{aligned}
-\left.\rho_{0}(0) \mathcal{P}_{\Gamma} \gamma_{0}\right|_{\Gamma} & =-\rho_{0}(0) \mathcal{P}_{\Gamma} \gamma_{0}\left[T u_{3}+A^{-1} f\right] \\
& =C_{0} u_{3}-\rho_{0}(0) \mathcal{P}_{\Gamma} \gamma_{0} A_{0}^{-1} f,
\end{aligned}
$$

where $C_{0}$ is defined in (24). We introduce also $\gamma_{n}$ the normal trace operator from $\mathcal{H}^{1}(\Omega)$ into $L^{2}(\Gamma): u_{3}=\left.u_{n}\right|_{\Gamma}=\gamma_{n} \boldsymbol{u}$.

Therefore, the equations of the small oscillation of the system (19) and (27) become the following for the unknown $\boldsymbol{u}$ and $f$ :

$$
\begin{aligned}
& \lambda^{2} \boldsymbol{u}-v \lambda A \boldsymbol{u}+\frac{1}{\rho} A T\left[\left(\rho-\rho_{0}(0)\right) g \gamma_{n} \boldsymbol{u}\right. \\
& \left.\quad+\lambda^{2}\left(C_{0} \gamma_{n} \boldsymbol{u}-\rho_{0}(0) \mathcal{P}_{\Gamma} \gamma_{0} A_{0}^{-1} f\right)\right]=0, \\
& f+\lambda^{2}\left(T_{0} \gamma_{n} \boldsymbol{u}+A_{0}^{-1} f\right)=0, \boldsymbol{u} \in \mathcal{J}_{0, \Sigma}^{1}(\Omega) ; f \in L_{\Omega_{0}}^{2}\left(\Omega_{0}\right) .
\end{aligned}
$$

We get equations the equations with bounded coefficient, by setting

$$
\boldsymbol{u}=A^{-1 / 2} \boldsymbol{U}, \quad \boldsymbol{U} \in \mathcal{J}_{0, \Sigma}^{1}(\Omega)
$$

and by applying $A^{-1 / 2}$ to the Eq. (28). We obtain

$$
\begin{aligned}
& \lambda^{2} A^{-1} \boldsymbol{U}-v \lambda \boldsymbol{U}+\frac{\rho-\rho_{0}(0)}{\rho} g A^{1 / 2} T \gamma_{n} A^{-1 / 2} \boldsymbol{U} \\
& +\frac{1}{\rho} \lambda^{2} A^{1 / 2} T C_{0} \gamma_{n} A^{-1 / 2} \boldsymbol{U} \\
& \quad-\frac{\rho_{0}(0)}{\rho} \lambda^{2} A^{1 / 2} T \mathcal{P}_{\Gamma} \gamma_{0} A_{0}^{-1} f=0,
\end{aligned}
$$

$$
\begin{aligned}
& \lambda^{2} T_{0} \gamma_{n} A^{-1 / 2} \boldsymbol{U}+\lambda^{2} A_{0}^{-1} f+f=0, \\
& \boldsymbol{U} \in \mathcal{J}_{0, \Sigma}^{1}(\Omega) ; \quad f \in L_{\Omega_{0}}^{2}\left(\Omega_{0}\right) .
\end{aligned}
$$

\subsection{Properties of the operators}

(a) The operators $\gamma_{n} A^{-1 / 2}$ (from $\mathcal{J}_{0, \Sigma}^{1}(\Omega)$ into $\tilde{L}^{2}(\Gamma)$ ) and $A^{1 / 2} T$ (from $\tilde{L}^{2}(\Gamma)$ ) into $\mathcal{J}_{0, \Sigma}^{1}(\Omega)$ ) are mutually adjoint and compact.

We have, by definition of $T$ :

$$
\left(\xi, \gamma_{n} \boldsymbol{u}\right)_{\tilde{L}^{2}(\Gamma)}=(T \xi, \boldsymbol{u})_{1}
$$

and then

$$
\begin{aligned}
& \left(\xi, \gamma_{n} \boldsymbol{u}\right)_{\tilde{L}^{2}(\Gamma)}=\left(A^{1 / 2} T \xi, A^{1 / 2} \boldsymbol{u}\right)_{\mathcal{J}_{0, \Sigma}(\Omega)} \\
& \text { or } \quad\left(\xi, \gamma_{n} A^{-1 / 2} \boldsymbol{U}\right)_{\tilde{L}^{2}(\Gamma)}=\left(A^{1 / 2} T \xi, \boldsymbol{U}\right)_{\mathcal{J}_{0, \Sigma}(\Omega)} .
\end{aligned}
$$

On the other hand, since $\gamma_{n}$ is compact, also $\gamma_{n} A^{-1 / 2}$ is and, by Schauder theorem, $A^{1 / 2} T$ is compact.

(b) The operators $T_{0}$ (from $\tilde{L}^{2}(\Gamma)$ into $L_{\Omega_{0}}^{2}\left(\Omega_{0}\right)$ ) and $-\rho_{0}(0) \mathcal{P}_{\Gamma} \gamma_{0} A_{0}^{-1}$ (from $L_{\Omega_{0}}^{2}\left(\Omega_{0}\right)$ into $\tilde{L}^{2}(\Gamma)$ ) are mutually adjoint and compact. We have

$$
\begin{aligned}
\left(T_{0} u_{3}, f\right)_{L_{\Omega_{0}}^{2}\left(\Omega_{0}\right)} & =\left(\Phi_{01}, A_{0} \Phi_{02}\right)_{L_{\Omega_{0}}^{2}\left(\Omega_{0}\right)} \\
& =\left(\Phi_{01}, \Phi_{02}\right)_{H_{\Omega_{0}}^{1}\left(\Omega_{0}\right)}, \\
\left(u_{3},-\rho_{0}(0) \mathcal{P}_{\Gamma} \gamma_{0} A_{0}^{-1} f\right)_{\tilde{L}^{2}(\Gamma)} & =-\left.\int_{\Gamma} \rho_{0}(0) u_{3} \Phi_{02}\right|_{\Gamma} d \Gamma .
\end{aligned}
$$

But we have $\tilde{\Phi}_{01}$ corresponding to the datum $\tilde{u}_{3}$ of the problem I:

$\int_{\Omega_{0}} \operatorname{grad} \Phi_{01} \cdot \operatorname{grad} \overline{\tilde{\Phi}}_{01} d \Omega_{0}=-\int_{\Gamma} \rho_{0}(0) u_{3} \mathcal{P}_{\Gamma} \gamma_{0} T_{0} \overline{\tilde{u}}_{3} d \Gamma$.

Replacing $\tilde{\Phi}_{01}$ arbitrary in $H_{\Omega_{0}}^{1}\left(\Omega_{0}\right)$ by $\Phi_{02}$ and then $T_{0} \tilde{u}_{3}$ by $\Phi_{02}$, we obtain 
$\int_{\Omega_{0}} \rho_{0} \operatorname{grad} \Phi_{01} \cdot \operatorname{grad} \bar{\Phi}_{02} d \Omega_{0}$

$=\int_{\Gamma} \rho_{0}(0) u_{3} \mathcal{P}_{\Gamma} \gamma_{0} \bar{\Phi}_{02} d \Gamma=-\left.\int_{\Gamma} \rho_{0}(0) u_{3} \bar{\Phi}_{02}\right|_{\Gamma} d \Gamma$,

or $\left(\Phi_{01}, \Phi_{02}\right)_{H_{\Omega_{0}}^{1}\left(\Omega_{0}\right)}=-\left.\int_{\Gamma} \rho_{0}(0) u_{3} \bar{\Phi}_{02}\right|_{\Gamma} d \Gamma$,

or, finally,

$\left(T_{0} u_{3}, f\right)_{L_{\Omega_{0}}^{2}\left(\Omega_{0}\right)}=\left(u_{3},-\rho_{0}(0) \mathcal{P}_{\Gamma} \gamma_{0} A_{0}^{-1} f\right)_{\tilde{L}^{2}(\Gamma)}$,

the compactness of all operators is a consequence of the compactness of $\gamma_{0}$ and the Schauder theorem.

(c) From the precedent results it follows that the operators

$K=-\rho_{0}(0) A^{1 / 2} T \mathcal{P}_{\Gamma} \gamma_{0} A_{0}^{-1}$

(from $L_{\Omega_{0}}^{2}\left(\Omega_{0}\right)$ into $\mathcal{J}_{0, \Sigma}(\Omega)$ ) and $T_{0} \gamma_{n} A^{-1 / 2}$ (from $\left.\mathcal{J}_{0, \Sigma}(\Omega)\right)$ into $L_{\Omega_{0}}^{2}\left(\Omega_{0}\right)$ ) are mutually adjoint and compact.

\subsection{Matricial form of the equations}

We set

$y=\left(\begin{array}{c}\boldsymbol{U} \\ f\end{array}\right) \in \mathcal{H} \overbrace{=}^{\text {def }} \mathcal{J}_{0, \Sigma}(\Omega) \oplus L_{\Omega_{0}}^{2}\left(\Omega_{0}\right)$,

the Eq. (30), multiplied by $\rho$, and (31) can be rewritten:

$$
\begin{aligned}
& \left\{\lambda^{2}\left(\begin{array}{cc}
\rho A^{-1}+A^{1 / 2} T C_{0} \gamma_{n} A^{-1 / 2} & K \\
T_{0} \gamma_{n} A^{-1 / 2} & A_{0}^{-1}
\end{array}\right)\right. \\
& -\lambda\left(\begin{array}{cc}
\mu I_{\mathcal{J}_{0, \Sigma}(\Omega)} & 0 \\
0 & 0
\end{array}\right) . \\
& \left.+\left(\begin{array}{cc}
\left(\rho-\rho_{0}(0)\right) g A^{1 / 2} T \gamma_{n} A^{-1 / 2} & 0 \\
0 & I_{L_{\Omega_{0}}^{2}\left(\Omega_{0}\right)}
\end{array}\right)\right\} y=0,
\end{aligned}
$$

where $K$ is defined by (32).

\section{First properties of the spectrum}

\subsection{Properties}

Proposition $1 \lambda=0$ is an eigenvalue with infinite multiplicity.
Proof Replacing $\lambda$ by 0 in the Eq. (34), we obtain $A^{1 / 2} T \gamma_{n} A^{-1 / 2} \boldsymbol{U}=0 ; \quad f=0$.

If $\boldsymbol{U} \neq 0$, we have

$$
\begin{aligned}
& 0=\left(A^{1 / 2} T \gamma_{n} A^{-1 / 2} \boldsymbol{U}, \boldsymbol{U}\right)_{\mathcal{J}_{0, \Sigma}(\Omega)} \\
& =\left\|\gamma_{n} A^{-1 / 2} \boldsymbol{U}\right\|_{\tilde{L}^{2}(\Gamma)}^{2} .
\end{aligned}
$$

Then $\boldsymbol{U} \in \operatorname{Ker}\left(\gamma_{n} A^{-1 / 2}\right)$ and the corresponding eigenspace is formed by the elements

$y=\left(\begin{array}{c}\boldsymbol{U} \in \operatorname{Ker}\left(\gamma_{n} A^{-1 / 2}\right) \\ f=0\end{array}\right)$.

Proposition 2 The spectrum is symmetric with respect to the real axis.

Proof The property of the spectrum is verified as the coefficients in (34) are self-adjoint.

\subsection{Existence of the spectrum}

Discarding $\lambda=0$, we multiply the Eq. (34) by $-\lambda^{-1}$, and we write the Eqs. (30), (31) in the form:

$$
\begin{aligned}
& \left\{\left(\begin{array}{cc}
\mu I_{\mathcal{J}_{0, \Sigma}(\Omega)} & 0 \\
0 & 0
\end{array}\right)-\lambda\left(\begin{array}{cc}
\rho A^{-1}+A^{1 / 2} T C_{0} \gamma_{n} A^{-1 / 2} & K \\
0 & 0
\end{array}\right)\right. \\
& -\lambda^{-1}\left(\begin{array}{cc}
\left(\rho-\rho_{0}(0)\right) g A^{1 / 2} T \gamma_{n} A^{-1 / 2} & 0 \\
0 & I_{L_{\Omega_{0}}^{2}\left(\Omega_{0}\right)}
\end{array}\right)+ \\
& \left.-\lambda\left(\begin{array}{cc}
0 & 0 \\
T_{0} \gamma_{n} A^{-1 / 2} & A_{0}^{-1}
\end{array}\right)\right\} y=0 .
\end{aligned}
$$

As the operator

$\mathcal{I}=\left(\begin{array}{cc}\mu I_{\mathcal{J}_{0, \Sigma}(\Omega)} & 0 \\ 0 & I_{L_{\Omega_{0}}^{2}\left(\Omega_{0}\right)}\end{array}\right)$

is self-adjoint and strongly positive, it has an inverse. Setting

$\mathcal{I}^{1 / 2} y=z \in \mathcal{H}$

where $\mathcal{H}$ is defined by (33), and applying $\mathcal{I}^{-1}$, we obtain 


$$
\begin{aligned}
& \mathcal{L}(z) \stackrel{\text { def }}{=}_{=}^{\text {I }}\left\{I_{\mathcal{H}}-\lambda \mathcal{I}^{-1 / 2}\left(\begin{array}{cc}
\rho A^{-1}+A^{1 / 2} T C_{0} \gamma_{n} A^{-1 / 2} & K \\
0 & 0
\end{array}\right) \mathcal{I}^{-1 / 2}\right. \\
& -\lambda^{-1} \mathcal{I}^{-1 / 2}\left(\begin{array}{cc}
\left(\rho-\rho_{0}(0)\right) g A^{1 / 2} T \gamma_{n} A^{-1 / 2} & 0 \\
0 & 0
\end{array}\right) \\
& \left.+\lambda^{2} \mathcal{I}^{-1 / 2}\left(\begin{array}{cc}
0 & 0 \\
T_{0} \gamma_{n} A^{-1 / 2} & A_{0}^{-1}
\end{array}\right) \mathcal{I}^{-1 / 2}\right\} z=0 .
\end{aligned}
$$

Except $I_{\mathcal{H}}$, all operators are compact and we have a Fredholm pencil, (see [3], p. 66).

We are going to prove that this pencil is regular, see [3], by showing that $\mathcal{L}(-1)$ has an inverse, where $\mathcal{L}(-1)$ is the value of the operator $\mathcal{L}$ for $\lambda=-1$. We can write,

$$
(\mathcal{L}(-1)(z), z)_{\mathcal{H}}=\|z\|_{\mathcal{H}}^{2}+\left(\mathcal{A}_{0} y, y\right)_{\mathcal{H}},
$$

with

$$
\begin{aligned}
\left(\mathcal{A}_{0} y, y\right)_{\mathcal{H}}= & \rho\left(A^{-1} \boldsymbol{U}, \boldsymbol{U}\right)_{\mathcal{J}_{0, \Sigma}} \\
& +\left(\rho-\rho_{0}(0)\right) g\left(A^{1 / 2} T \gamma_{n} A^{-1 / 2} \boldsymbol{U}, \boldsymbol{U}\right)_{\mathcal{J}_{0, \Sigma}} \\
& +\left(A^{1 / 2} T C_{0} \gamma_{n} A^{-1 / 2} \boldsymbol{U}, \boldsymbol{U}\right)_{\mathcal{J}_{0, \Sigma}}+\left(A_{0}^{-1} f, f\right)_{L_{\Omega_{0}}^{2}\left(\Omega_{0}\right)} \\
& +2 \mathcal{R}\left(T_{0} \gamma_{n} A^{-1 / 2} \boldsymbol{U}, f\right)_{L_{\Omega_{0}}^{2}\left(\Omega_{0}\right)}
\end{aligned}
$$

We have:

$$
\begin{aligned}
& \left(A^{-1} \boldsymbol{U}, \boldsymbol{U}\right)_{\mathcal{J}_{0, \Sigma}} \geq 0, \\
& \left(A^{1 / 2} T \gamma_{n} A^{-1 / 2} \boldsymbol{U}, \boldsymbol{U}\right)_{\mathcal{J}_{0, \Sigma}}=\left\|\gamma_{n} A^{-1 / 2} \boldsymbol{U}\right\|_{\tilde{L}^{2}(\Gamma)} \geq 0
\end{aligned}
$$

and

$$
\begin{aligned}
& \left(\rho-\rho_{0}(0)\right) g\left(A^{1 / 2} T \gamma_{n} A^{-1 / 2} \boldsymbol{U}, \boldsymbol{U}\right)_{\mathcal{J}_{0, \Sigma}}+\left(A_{0}^{-1} f, f\right)_{L_{\Omega_{0}}^{2}\left(\Omega_{0}\right)} \\
& +2 \mathcal{R}\left(T_{0} \gamma_{n} A^{-1 / 2} \boldsymbol{U}, f\right)_{L_{\Omega_{0}}^{2}\left(\Omega_{0}\right)} \\
& \quad=\left\|\Phi_{01}+\Phi_{02}\right\|_{H_{\Omega_{0}}^{1}\left(\Omega_{0}\right)}^{2} \geq 0,
\end{aligned}
$$

where $\Phi_{01}$ and $\Phi_{02}$ are defined in (23) and (26), respectively. Finally, we obtain

$$
(\mathcal{L}(-1)(z), z)_{\mathcal{H}} \geq\|z\|_{\mathcal{H}}^{2}, \quad \forall z \in \mathcal{H}
$$

as $\mathcal{L}(-1)$, self-adjoint and strongly positive, it has a bounded inverse.

So, (see [3], p. 66), we have the following:

Theorem 3 The Fredholm pencil $\mathcal{L} t(z)$ is regular in the domain $\mathbb{C} \backslash\{0\} \backslash\{\infty\}$. Its spectrum consists of isolated points that are eignvalues and its accumulation points may be only 0 and $\infty$. All points of the spectrum are eigenvalues. The eigenelements have finite multiplicities.

\section{Stability of the system liquid-gas}

We shall prove that the eigenvalues are located in the half-plane $\mathcal{R} \lambda \geq 0$.

The Eq. (34) divided by $-\lambda$ takes the form

$$
\left(\mathcal{I}_{0}-\lambda \mathcal{A}-\lambda^{-1} \mathcal{B}\right) y=0
$$

with

$$
\begin{aligned}
\mathcal{I}_{0} & =\left(\begin{array}{cc}
\mu I_{\mathcal{J}_{0, \Sigma}(\Omega)} & 0 \\
0 & 0
\end{array}\right), \\
\mathcal{A} & =\left(\begin{array}{cc}
\rho A^{-1}+A^{1 / 2} T C_{0} \gamma_{n} A^{-1 / 2} & K \\
T_{0} \gamma_{n} A^{-1 / 2} & A_{0}^{-1}
\end{array}\right), \\
\mathcal{B} & =\left(\begin{array}{cc}
\left(\rho-\rho_{0}(0)\right) g A^{1 / 2} \gamma_{n} A^{-1 / 2} & 0 \\
0 & I_{L_{\Omega_{0}}^{2}\left(\Omega_{0}\right)}
\end{array}\right) ;
\end{aligned}
$$

$\mathcal{A}$ and $\mathcal{B}$ are self-adjoint, $\mathcal{A}$ is compact, but $\mathcal{B}$ is not.

Taking the scalar product by $y$ in $\mathcal{H}$, we deduce

$$
\mu\|\boldsymbol{U}\|_{\mathcal{J}_{0, \Sigma}(\Omega)}^{2}=\lambda(\mathcal{A} y, y)_{\mathcal{H}}+\lambda^{-1}(\mathcal{B} y, y)_{\mathcal{H}}
$$

and then

$$
\mu\|\boldsymbol{U}\|_{\mathcal{J}_{0, \Sigma}(\Omega)}^{2}=\mathcal{R} \lambda\left[(\mathcal{A} y, y)_{\mathcal{H}}+\frac{1}{|\lambda|^{2}}(\mathcal{B} y, y)_{\mathcal{H}}\right] .
$$

By direct calculations, we have

$$
\begin{aligned}
& (\mathcal{B} y, y)_{\mathcal{H}}=\left[\rho-\rho_{(0)}\right] g\left\|\gamma_{n} A^{-1} \boldsymbol{U}\right\|_{L^{2}(\Gamma)}^{2}+\|f\|_{L_{\Omega_{0}}^{2}\left(\Omega_{0}\right)}^{2} \geq 0, \\
& (\mathcal{A} y, y)_{\mathcal{H}}=\rho\left(A^{-1} \boldsymbol{U}, \boldsymbol{U}\right)_{\mathcal{J}_{0, \Sigma}}+\left\|\Phi_{01}+\Phi_{02}\right\|_{H_{\Omega_{0}}^{1}\left(\Omega_{0}\right)}^{2} \geq 0 ;
\end{aligned}
$$

so that

$\mathcal{R} \lambda \geq 0$.

Consequently, the eigenvalues are located in the right plane $\mathcal{R} \lambda \geq 0$.

We are going to prove that $\mathcal{R} \lambda>0$. We can have $\mathcal{R} \lambda=0$ (then $\lambda=i \gamma, \gamma$ real number) if $\boldsymbol{U}=0$. Then, the Eqs. (30) and (31) become

$$
A^{1 / 2} T \mathcal{P}_{\Gamma} \gamma_{0} A_{0}^{-1} f=0 ; A_{0} f=\gamma^{2} f .
$$

Let us denote by $\omega_{j}^{2}, j=1,2, \ldots$ the eigenvalues of 
$A_{0}$. If $\gamma \neq \pm \omega_{j}$, the second equation of (36) gives $f=$ 0 , so that $\lambda=i \gamma$ cannot be eigenvalues of the problem. If $\gamma= \pm \omega_{j}$, the second equation of (36) is verified by $f=f_{j}$, eigenfunction corresponding to $\omega_{j}^{2}$. But $f_{j}$ depends on the gas and $A, T$ on the liquid, so that the first equation of (36)

$$
A^{1 / 2} T \mathcal{P}_{\Gamma} \gamma_{0} A_{0}^{-1} f_{j}=0
$$

is not satisfied, in general. As $\mathcal{R} \lambda>0$, we have proved the following:

Theorem 4 The system liquid-gas is stable in linear approsimation.

\section{Existence of a sets of positive real eigenvalues}

The Eq. (31) can be written as

$$
\left(I_{L_{\Omega_{0}}^{2}\left(\Omega_{0}\right)}+\lambda^{2} A_{0}^{-1}\right) f=-\lambda^{2} T_{0} \gamma_{n} A^{-1 / 2} \boldsymbol{U}
$$

If $|\lambda|$ is sufficiently small, setting

$$
m(\lambda)=I_{L_{\Omega_{0}}^{2}\left(\Omega_{0}\right)}+\lambda^{2} A_{0}^{-1},
$$

it has an inverse $m(\lambda)^{-1}$ that is self-adjoint and holomorphic in the vicinity of $\lambda=0$. Consequently, we have

$f=-\lambda^{2} m(\lambda)^{-1} T_{0} \gamma_{n} A^{-1 / 2} \boldsymbol{U}$.

Carrying out the Eq. (30), we obtain

$$
\begin{aligned}
& \hat{\mathcal{L}}(\lambda) \boldsymbol{U}_{=}^{\text {def }}\left[\lambda^{2} A^{-1}-v \lambda I_{\mathcal{J}_{0, \Sigma}(\Omega)}\right. \\
& \quad+\frac{\left(\rho-\rho_{0}(0)\right) g}{\rho} A^{1 / 2} T \gamma_{n} A^{-1 / 2}+\lambda^{2} A^{1 / 2} T C_{0} \gamma_{n} A^{-1 / 2} . \\
& \left.\quad-\frac{\rho_{0}(0)}{\rho} \lambda^{4} K m(\lambda)^{-1} T_{0} \gamma_{n} A^{-1 / 2}\right] \boldsymbol{U}=0 .
\end{aligned}
$$

Obviously, $\hat{\mathcal{L}}(\lambda)$ is holomorphic in the vicinity of $\lambda=$ 0 and self-adjoint. Moreover

$$
\hat{\mathcal{L}}(0)=\frac{\left(\rho-\rho_{0}(0)\right) g}{\rho} A^{1 / 2} T \gamma_{n} A^{-1 / 2} ;
$$

$\hat{\mathcal{L}}(0)$ is compact, and not negative, indeed

$$
(\hat{\mathcal{L}}(0) \boldsymbol{U}, \boldsymbol{U})_{\mathcal{J}_{0, \Sigma}(\Omega)}=\frac{\left(\rho-\rho_{0}(0)\right) g}{\rho}\left\|\gamma_{n} A^{-1 / 2} \boldsymbol{U}\right\|_{\tilde{L}^{2}(\Gamma)}^{2} \geq 0,
$$

but it is equal to zero for $\boldsymbol{U} \in \operatorname{Ker}\left(A^{-1 / 2} \gamma_{n}\right)$.

Moreover, it results

$\hat{\mathcal{L}}^{\prime}(0)=-v I_{\mathcal{J}_{0, \Sigma}(\Omega)}$

and it is strongly negative.

Consequently, using a well-known theorem ([3], p. 74), we have the following:

Theorem 5 For each $\varepsilon>0$ sufficiently small, there exists in $]-\varepsilon, \varepsilon\left[\right.$ a set of positive real eigenvalues $\lambda_{n}^{0}$ having zero as point of accumulation. The corresponding eigenelements $\boldsymbol{U}_{n}^{0}$ and an orthogonal basis of $\operatorname{Ker}\left(\gamma_{n} A^{-1 / 2}\right)$ form a Riesz basis in a subspace of $\mathcal{J}_{0, \Sigma}(\Omega)$ with finite defect.

\section{Reduction to a Krein-Langer pencil}

The Eq. (35) can be written as

$$
\left(\lambda^{2} \mathcal{A}-\lambda \mathcal{I}_{0}+\mathcal{B}\right) y=0, \quad y \in \mathcal{H},
$$

where $\mathcal{H}$ is defined in (33). Setting $\lambda=-\lambda^{\prime}-k, k$ positive and constant, we obtain

$$
\left[\lambda^{\prime 2} \mathcal{A}+\lambda^{\prime}\left(\mathcal{I}_{0}+2 k \mathcal{A}\right)+\left(k^{2} \mathcal{A}+k \mathcal{I}_{0}+\mathcal{B}\right)\right] y=0 .
$$

By direct calculations, we have

$$
\left(\left(k^{2} \mathcal{A}+k \mathcal{I}_{0}+\mathcal{B}\right) y, y\right)_{\mathcal{H}} \geq k \mu\|\boldsymbol{U}\|_{\mathcal{J}_{0, \Sigma}(\Omega)}^{2}+\|f\|_{L_{\Omega_{0}}^{2}\left(\Omega_{0}\right)}^{2},
$$

so that the operator

$$
\mathcal{F} \overbrace{=}^{\text {def }} k^{2} \mathcal{A}+k \mathcal{I}_{0}+\mathcal{B}
$$

is bounded, self-adjoint and strongly positive, then it has an inverse. Putting

$\mathcal{F}^{1 / 2} y=\hat{z} \in \mathcal{H}$,

we obtain the equation

$$
\left(\lambda^{\prime 2} \mathcal{C}_{0}+\lambda^{\prime} \mathcal{B}_{0}+I_{\mathcal{H}}\right) \hat{z}=0
$$

with 


$$
\begin{aligned}
\mathcal{C}_{0} & =\mathcal{F}^{-1 / 2} \mathcal{A} \mathcal{F}^{-1 / 2} \text { and } \\
\mathcal{B}_{0} & =\mathcal{F}^{-1 / 2}\left(\mathcal{I}_{0}+2 k \mathcal{A}\right) \mathcal{F}^{-1 / 2}
\end{aligned}
$$

We recognize that the operator $\mathcal{C}_{0}$ is self-adjoint, compact like $\mathcal{A}$ and positive definite; indeed

$$
\left(\mathcal{C}_{0} \hat{z}, \hat{z}\right)_{\mathcal{H}}=(\mathcal{A} y, y)_{\mathcal{H}} \geq 0,
$$

and equal to zero only for $y=0$.

The operator $\mathcal{B}_{0}$ is self-adjoint and positive definite, as

$$
\left(\mathcal{B}_{0} \hat{z}, \hat{z}\right)_{\mathcal{H}}=\mu\|\boldsymbol{U}\|_{\mathcal{J}_{0, \Sigma}(\Omega)}^{2}+2 k(\mathcal{A} y, y)_{\mathcal{H}}
$$

but it is not compact.

Therefore, for each positive real $k$, the pencil

$$
\mathcal{L}_{0}\left(\lambda^{\prime}\right)=\lambda^{\prime 2} \mathcal{C}_{0}+\lambda^{\prime} \mathcal{B}_{0}+I_{\mathcal{H}}
$$

is a Krein-Langer operator pencil.

The theoretical study of the operator pencil can be found in the book ([8], pp. 295-309). For our problem, we restrict ourselves to the following new result:

Theorem 6 Since $\mathcal{B}_{0}$ is self-adjoint, the nonreal eigenvalues can have as point of accumulation only the infinity.

Acknowledgements For the first author, this research is in the framework of by GNFM of Miur (Italy).

\section{Compliance with ethical standards}

Conflict of interest The authors declare that they have no conflict of interest.

\section{References}

1. Moiseyev NN (1952) About the oscillations of an ideal incompressible liquid in a container. In: Doklady AN SSSR, vol 85, pp 1-20 (in Russian)

2. Moiseyev NN, Rumiantsev VV (1968) Dynamic stability of bodies containing fluid. Springer, Berlin

3. Kopachevskii ND, Krein SG (2001) Operator approach in linear problems of hydrodynamics: self-adjoint problems for an ideal fluid, vol 1. Birkhäuser-Verlag, Basel

4. Morand HJ-P, Ohayon R (1992) Interactions fluides-structures. Masson, Paris

5. Kopachevskii ND, Krein SG (2003) Operator approach in linear problems of hydrodynamics: self-adjoint problems for viscous fluids, vol 2. Birkhäuser-Verlag, Basel

6. Wronskii BM (1986) "Oscillations of the system fluid-gas" in a bounded region. In: Preprint n. 1811, Simferopol, p 26 (in Russian)

7. Essaouni H, El Bakkali L, Capodanno P (2010) Analysis of the small oscillations of a heavy almost-homogeneous liquid-gas system. Mech Res Commun (MRC) 37:337-340

8. Gomberg IC, Kein MG (1971) Introduction ä la théorie des opérateurs linéaires non-autodjoints dans un espace hilbertien. Dunod, Paris

9. Sanchez-Hubert J, Sanchez-Palencia E (1989) Vibrarion and coupling of continuous systems-Asymptotic methods. Springer, Berlin

10. Lions JL (1961) Equations differentielle opërationelles et problëmes aux limites. Springer, Berlin 